\title{
Circuit-level stability and bifurcation analysis of non-foster circuits
}

\author{
Almudena Suárez, Franco Ramírez \\ University of Cantabria, Avda. Los Castros sn, 39005, Santander, Spain
}

\begin{abstract}
An in-depth stability analysis of a typical non-Foster matching network is presented. The investigation is carried out at two levels: considering an ideal implementation of the negative impedance inverter (NIC) and using detailed circuit-level descriptions of all its active and passive components. The ideal NIC model will enable an analytical derivation of the characteristic system and the system poles, which will provide insight into the main instability mechanisms in these configurations. A good qualitative agreement is obtained with the circuit-level analyses, based on pole-zero identification and bifurcation detection methods. The impact of significant parameters, such as the biasing resistors or the value of the reactive component to be negated, is investigated in detail. A circuit-level methodology is proposed to obtain the stability boundaries and margins in an efficient and rigorous manner. For illustration, a non-Foster circuit based on a NIC has been manufactured and measured, obtaining very good agreement with the analysis results.

Index Terms-Non-Foster network, stability, bifurcation.
\end{abstract}

\section{INTRODUCTION}

Non-Foster circuits enable a broadband impedance matching of antennas and other system components [1]-[4], which relies on the implementation of negative capacitors or inductors. These are used to cancel the reactive effects in the element to be matched, usually an electrically small antenna. The implementation is carried out through transistor-based negative impedance converters (NICs) [1]-[3]. In [2] it is demonstrated that the NIC is a potentially unstable two-port network. As derived from its $h$-parameter matrix [3], any practical NIC is always short-circuit (SC) stable and open-circuit (OC) unstable at one port (that is, when terminating that port in SC and OC, respectively) and $\mathrm{OC}$ stable and SC unstable at the other port. As a result, the matching ability of the non-Foster network will be limited by its possible unstable behavior.

Several previous works have addressed the stability analysis of non-Foster circuits [3]-[6]. Some works rely on simplified equivalent networks of the loaded NIC [2], represented as a negative capacitor (or inductor), which is unrealistic. With this simple model one concludes that for an exact cancellation of the antenna reactance, the circuit operates at a bifurcation condition [7]. When considering the transistor-based NIC, most of the stability analyses presented are based on time-domain integration, applying a Gaussian pulse [5], or on the Nyquist criterion [4], [6]. The time-domain analysis may be unreliable since, as demonstrated here, transients can be very long in NIC circuits. In other methods, the Nyquist criterion is applied to the open-loop gain [4], [6]. When correctly implemented, this criterion is accurate and complete, though it enables little insight into the instability mechanism. The number of encirclements of the Nyquist plot around $-1+j 0$ provides the difference between the number of zeroes $(Z)$ and poles $(P)$ of the open-loop gain, located on the right hand-side of the complex plane (RHS), $N=Z-P$, where $Z$ agrees with the number of system poles. The analyzed function should not contain any RHS poles as otherwise $Z$ is undetermined. This is ensured through the application of the Normalized Determinant Function [6]. However, this function requires access to the intrinsic terminals of all the active devices and cannot be used with black-box models. Recently, pole-zero identification [8] has been applied to non-Foster circuits [9], presenting pole-zero maps for particular circuitcomponent values, without a detailed investigation of the instability phenomena.

In this work an in-depth stability analysis of a typical nonFoster monopole antenna is presented. The instability mechanisms are studied analytically and validated through pole-zero identification [8]. After a derivation of the bifurcation phenomena [7], a new method is presented to obtain the instability boundaries in an efficient manner, avoiding multiple and lengthy individual stability analyses versus the element values.

\section{ANALYTICAL STABILITY ANALYSIS}

The stability analysis will be illustrated through application to a non-Foster circuit based on the Yanigisawa configuration [Fig. 1(a)], implemented with bipolar transistors. The NIC action derives from the fact that, under an approximate transistor model, with the transconductance $g_{m}$, the input impedance of an isolated pair of cross-coupled transistors, terminated in the impedance $z$ (connected between the two collector terminals $v_{c 1}$, $v_{c 2}$, as shown in Fig. 1(a]) is $z_{i n}=2 / g_{m}-z$. The resistive term $\left(2 / g_{m}\right)$ is compensated [3] with an additional series resistor $R_{n}$, which can be absorbed in $z$. For an antenna impedance $z_{a}$ modeled as a series connection of a resistor $R_{a}$ and a capacitor $c_{a}$ [2]-[3], the NIC is used to obtain a negative capacitance and, thus, match the antenna to the receiver. Fig. 1(c) provides the simplified schematic for the analytical study. For better insight, the transistor capacitive elements are neglected. The circuit is governed by the characteristic system:

$$
\begin{aligned}
& g_{m} v_{1}+\left(G_{C}+y\right) v_{c 1}-y v_{c 2}+G_{B} v_{2}=0 \\
& g_{m} v_{2}+\left(G_{C}+y\right) v_{c 2}-y v_{c 1}+G_{B} v_{1}=0 \\
& v_{c 1}=\left(z_{a}\left(g_{m}+G_{B}\right)+\left[R_{B}+1 /\left(c_{d c} s\right)\right] G_{B}\right) v_{2} \\
& v_{c 2}=\left(z_{r e c}\left(g_{m}+G_{B}\right)+\left[R_{B}+1 /\left(c_{d c} s\right)\right] G_{B}\right) v_{1}
\end{aligned}
$$


where $y=z^{-1}, c_{d c}$ is the dc-blocking capacitor, $R_{C}=G_{C}^{-1}$ is the collector bias resistor, $R_{B}=G_{B}{ }^{-1}$ is the base resistor and $z_{\text {rec }}$ is the receiver impedance. In this investigation, two different instability problems have been identified, associated with the transistor cross-coupling and with the NIC action.

To highlight the problem with the transistor cross-coupling, the elements $y, z_{r e c}$ and $z_{a}$ will be eliminated, considering an $\mathrm{OC}$ between $v_{c 1}$ and $v_{c 2}(y=0)$, and SC terminations $\mathrm{z}_{r e c}=0$ and $z_{a}=0$ [Fig. 1(d)]. System (1) becomes:

$$
\begin{aligned}
& g_{m} v_{1}+\left[G_{B}+G_{C}+G_{C} G_{B} /\left(c_{d c} s\right)\right] v_{2}=0 \\
& {\left[G_{B}+G_{C}+G_{C} G_{B} /\left(c_{d c} s\right)\right] v_{1}+g_{m} v_{2}=0}
\end{aligned}
$$

Solving the system characteristic determinant, one obtains two real poles, given by:

$$
\begin{aligned}
& p_{1}=-G_{B} G_{C} /\left[\left(G_{C}+G_{B}-g_{m}\right) c_{d c}\right] \\
& p_{2}=-G_{B} G_{C} /\left[\left(G_{C}+G_{B}+g_{m}\right) c_{d c}\right]
\end{aligned}
$$

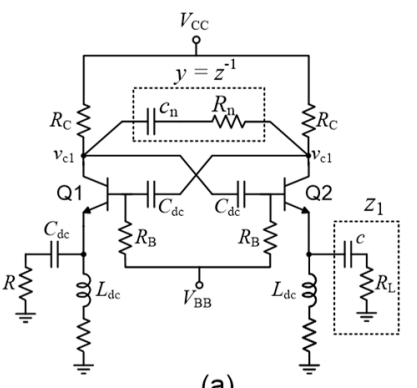

(a)

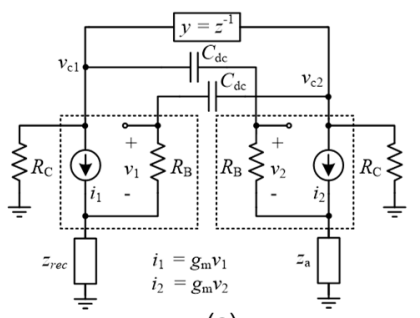

(c)

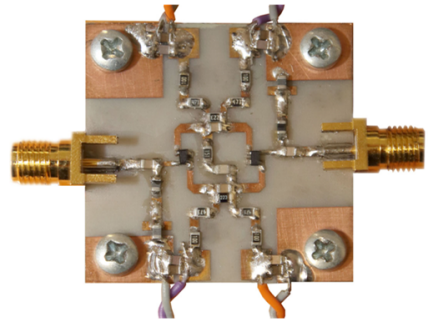

(b)

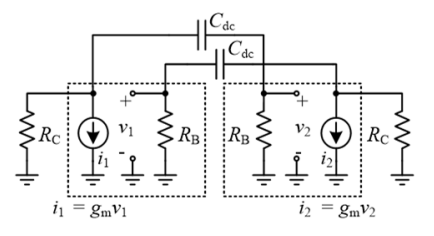

(d)
Fig. 1 Non-Foster circuit. (a) Realistic circuit. (b) Photograph of the implemented NIC converter. The prototype is based on Infineon BFP405 BJT transistors on RO3003 substrate $\left(\varepsilon_{\mathrm{r}}=3, \mathrm{~h}=0.75 \mathrm{~mm}\right)$. (c) Simplified description. (d) Simplified circuit to analyze the cross-coupling effects.

The stability condition is $G_{C}+G_{B}-g_{m}>0$, which imposes a limit to the transistor gain. Fig. 2(a) presents the variation of the poles versus $R_{C}$. The results are compared with a pole-zero analysis [8] of the complete circuit in Fig. 1(a), using realistic models of the transistor devices. At the instability boundary, $p_{1}$ in (3) tends to infinity, which is an awkward situation, resulting from the over-simplified model. In the circuit-level analysis, the two real poles merge into a pair of complex conjugate poles about the stability limit (horizontal axis in Fig. 2(a)], which is due to a Hopf bifurcation [7]. In a Hopf bifurcation, a pair of complex conjugate poles crosses the imaginary axis, giving rise to the onset or extinction of an oscillatory solution. Some applications where Hopf bifurcations are commonly observed include power amplifiers, microwave oscillators, etc. [10-12].

Both (3) and the circuit-level analysis predict the same bifurcation point. In the two cases there is a pole $\left[p_{2}\right.$ in (3)] very close to zero, associated with a slow time scale. In the unstable region, the circuit behaves as a relaxation oscillator. To investigate this, the function $i(v)=g_{m} v+g_{m 3} v^{3}$, with $g_{m 3}<0$, is considered in Fig. 1(d), to calculate the steady-state oscillation. The circuit is governed by the differential equation system:

$\frac{d v_{1}}{d t}=\frac{-G_{C} G_{B}}{c_{d c}\left[g_{m}+3 g_{m 3} v_{1}^{2}(t)\right]} v_{2}(t) ; \frac{d v_{2}}{d t}=\frac{-G_{C} G_{B}}{c_{d c}\left[g_{m}+3 g_{m 3} v_{2}^{2}(t)\right]} v_{1}(t)$

For $v_{2}(t)<0$ and $d i_{1} / d v_{1}=g_{m}+3 g_{m 3} v_{1}^{2}(t)<0$, due to a large $\left|v_{1}(t)\right|$, there is a negative slope in $d v_{1}(t) / d t$, until the denominator becomes zero and the slope tends to infinity. Then, $v_{2}(\mathrm{t})>0$ and $d v_{1}(t) / d t>0$ (note that the denominator depends on $\left|v_{1}(t)\right|$ ), since the opposite is true for $d v_{2}(t) / d t$ due to the circuit symmetry. Fig. 2(c) presents the oscillation simulated at circuitlevel oscillation and the measured oscillation.

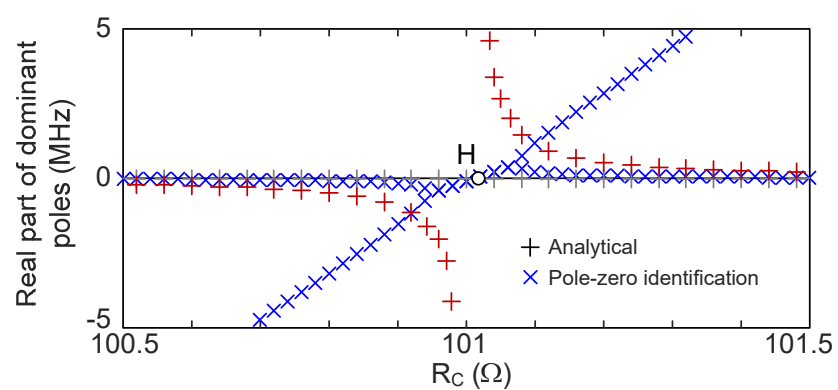

(a)

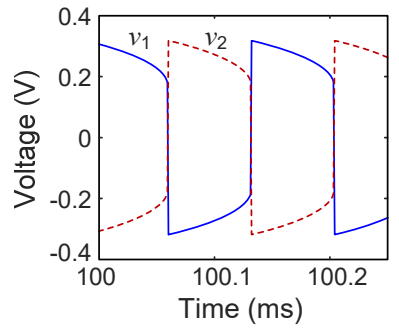

(b)

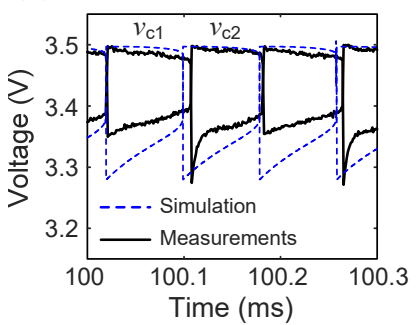

(c)
Fig. 2 Instability due to the transistor cross-coupling. (a) Comparison of analytical and circuit-level stability analyses vs. $R_{C}$. (b) Simplified-model relaxation oscillation. (c) Simulated and measured circuit-level oscillation,

To emphasize the instability due to the NIC action, the largevalue $R_{B}$ and the $c_{d c}$ capacitors are neglected, focusing on $z, z_{a}$ and $z_{\text {rec }}$. The characteristic system (1) simplifies to:

$$
\begin{array}{r}
{\left[g_{m}-y\left(z_{r e c} g_{m}+1\right)\right] v_{1}+\left(G_{c}+y\right)\left(z_{a} g_{m}+1\right) v_{2}=0} \\
\left(G_{c}+y\right)\left(z_{r e c} g_{m}+1\right) v_{1}+\left[g_{m}-y\left(z_{a} g_{m}+1\right)\right] v_{2}=0
\end{array}
$$

with the following characteristic determinant: 


$$
\left(A+B z_{a}\right) y+D+E z_{a}=0
$$

where:

$$
\begin{aligned}
& A=2 G_{c}+2 g_{m}\left(1+G_{c} R\right)+R g_{m}^{2} ; B=g_{m}^{2}+2 g_{m} G_{c}\left(1+R g_{m}\right) \\
& D=-g_{m}^{2}+G_{c}^{2}\left(1+R g_{m}\right) ; E=(1+R) g_{m} G_{c}^{2}
\end{aligned}
$$

Next, $z, z_{a}$ and $z_{\text {rec }}$ are particularized to their usual values:

$$
z^{-1}=y=c_{n} s ; \quad z_{a}=R_{a}+1 /\left(c_{a} s\right) ; \quad z_{\text {rec }}=R=50 \Omega
$$

where the compensation resistor $R_{n}$ is omitted to emphasize the effect of $c_{n}$. Calculating the characteristic determinant:

$$
\left(A+B R_{L}\right) c c_{n} s^{2}+\left(B c_{n}+D c+E R_{L} c\right) s+E=0
$$

All the quantities in (8) are positive definite except $D$ and the stability properties will depend on $g_{m}$. Fig. 3 presents the evolution of the real part of the two poles versus $c_{n}$, for $c_{a}=5 \mathrm{pF}$. In the lower $c_{n}$ range, the circuit is unstable, which is consistent with the fact that the negative capacitance due to the NIC dominates the positive one, as the two are ideally in a series connection. The two real poles merge into a pair of complex-conjugate poles at $c_{n}=1.75 \mathrm{pF}$ and cross to the left-hand-side of the complex plane (LHS) at $c_{n}=2.9 \mathrm{pF}$ in an inverse Hopf bifurcation. From inspection of (8), the only way to obtain a real pole at zero is through fulfillment of $E=0$, which is not possible [see (6)]. Thus, the stability boundary must correspond to a Hopf locus [7]. Results obtained with pole-zero identification of the full circuit are nearly overlapped. For most $c_{n}$ values in the unstable region, there are two positive real poles, associated with relaxation oscillations.

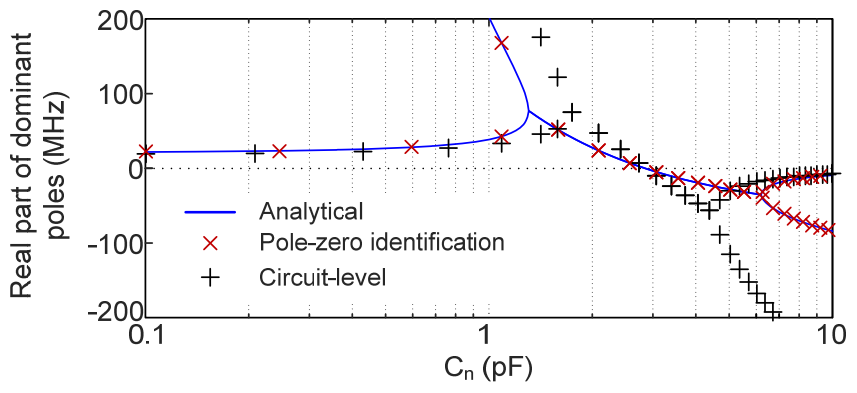

Fig. 3 Real part of the poles vs. $c_{n}$, for $c_{a}=5 \mathrm{pF}$. Analytical and circuit-level results. The former are also validated with pole-zero identification.

\section{HOPF BIFURCATION LOCUS}

The Hopf-bifurcation locus associated with the impedance to be negated $z$ can be calculated with an efficient method, directly applicable in commercial software. Two properties are taken into account: when a circuit oscillates, the total admittance function is equal to zero at all the circuit nodes and, at the Hopf bifurcation, this condition is fulfilled for oscillation amplitude tending to zero [7]. To obtain the Hopf locus, the impedance $z$ (to be negated) will be suppressed, performing a small-signal frequency sweep and calculating the total impedance $Z_{\text {in }}$ seen between the two nodes where $z$ should be connected. Then, the resistor and capacitor at the oscillation boundary will be those giving rise to the impedance $-Z_{i n}=R+1 /\left(j c_{n} \omega\right)$. This provides:

$$
R_{n}=-\operatorname{Re}\left(Z_{\text {in }}\right) ; \quad c_{n}=-1 /\left[\operatorname{Im}\left(Z_{\text {in }}\right) \omega\right]
$$

Any negative values of $R_{n}$ or $c_{n}$ should be discarded. Using (9) in the full circuit description of Fig. 1(a), one obtains the locus in Fig. 4. Inside the locus, the critical pair of complexconjugate poles is on the LHS. The Hopf locus has been validated with pole-zero identification. The evolution of the real part of the complex-conjugate poles is traced versus $c_{n}$ for two different $R_{n}$ values [indicated in Fig. 3(a)] in Fig. 4(b). To reinforce the circuit stability, the coupling capacitor $c_{d c}$ has been reduced from $100 \mathrm{nF}$ to $15 \mathrm{nF}$. After this modification, there are no solutions of the Hopf locus with $R_{n}>0$ [Fig. 4(a)]. All the points in the locus enclosing the region with RHS poles fulfill $R_{n}<0$. Fig. 5 presents the input reflection coefficient measured in the bandwidth $1 \mathrm{MHz}$ to $1 \mathrm{GHz}$, which demonstrates the stable non-Foster matching.

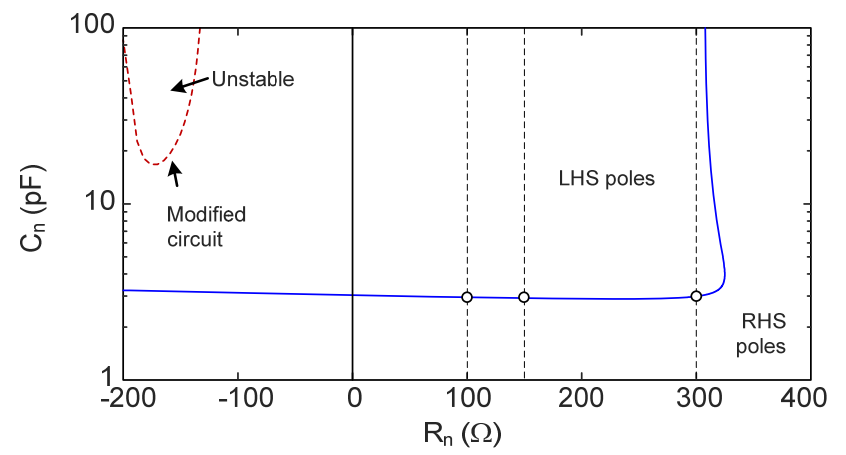

(a)

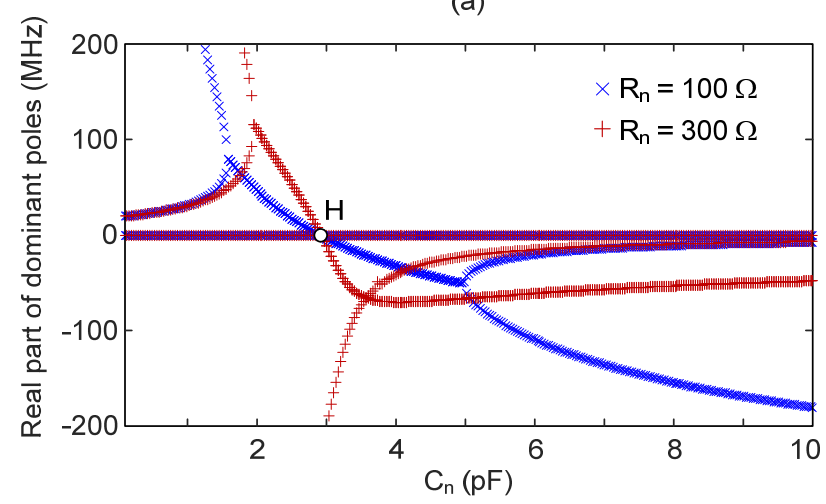

(b)

Fig. 4 Hopf bifurcation loci with $c_{a}=5 \mathrm{pF}$. (a) Loci for the original modified designs. (b) Validation of the Hopf locus of the original design for two different values of $R_{n}$. The real part of the poles has been traced versus $c_{n}$. 


\section{CONCLUSION}

An in-depth investigation of instability phenomena in nonFoster circuits has been presented. Instabilities arising from the transistor cross-coupling and from the NIC action have been identified, with the instability boundary being due to a Hopf bifurcation in all cases. A new methodology has been presented for an efficient prediction of the oscillation boundaries, directly applicable in commercial software, which avoids multiple and lengthy individual stability analyses versus the circuit elements.

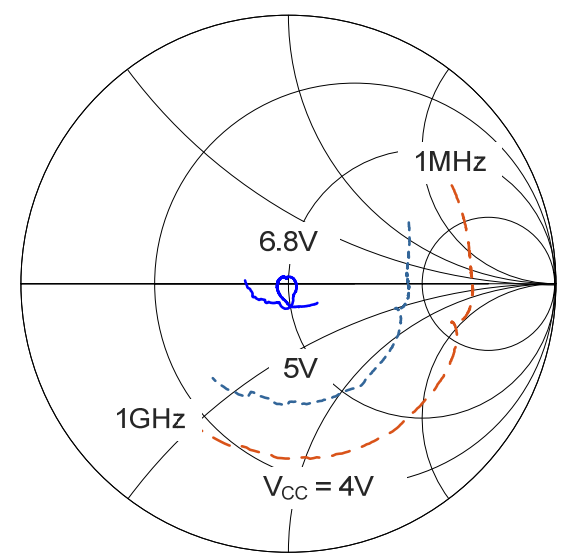

(a)

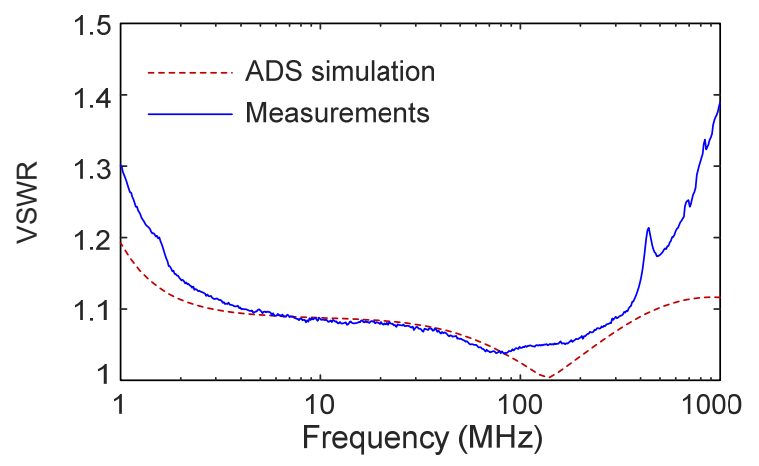

(b)

Fig. 5 Experimental matching from $1 \mathrm{MHz}$ to $1 \mathrm{GHz}$. (a) $S_{11}$ measured for different biasing voltages. (b) Simulated and measured VSWR for $\mathrm{V}_{\mathrm{cc}}=6.8 \mathrm{~V}$.

\section{ACKNOWLEDGMENT}

This work has been funded by the Spanish Government under contract TEC2014-60283-C3-1-R, the European Regional Development Fund (ERDF/FEDER) and the Parliament of Cantabria (12.JP02.64069). The authors would like to thank S. Pana, University of Cantabria, for her assistance with the manufacturing process.

\section{REFERENCES}

[1] S. E. Sussman-Fort, R. M. Rudish, "Non-Foster Impedance Matching of Electrically-Small Antennas," in IEEE Trans. Antennas Propag., vol. 57, no. 8, pp. 2230-2241, Aug., 2009.

[2] C. R. White, J. S. Colburn, R. G. Nagele, "A Non-Foster VHF Monopole Antenna," IEEE Antennas Wireless Propag. Lett., vol. 11, pp. 584-587, 2012.

[3] C. K. Kuo, "Realization of negative-immittance converters and negative resistances with controlled sources," PhD Thesis, Georgia Institute of Technology, December, 1967

[4] H. Mirzaei, G. V. Eleftheriades, "A Resonant Printed Monopole Antenna with an Embedded Non-Foster Matching Network," in IEEE Trans. Antennas Propag., vol. 61, no. 11, pp. 5363-5371, Nov. 2013.

[5] A. M. Elfrgani, R. G. Rojas, "Successful realization of Non-Foster circuits for wide-band antenna applications," 2015 IEEE MTT-S Int. Microw. Symp., Phoenix, AZ, 2015, pp. 1-4.

[6] S. D. Stearns, "Circuit stability theory for non-Foster circuits," 2013 IEEE MTT-S Int. Microw. Symp., Seattle, WA, 2013, pp. 1-3.

[7] A. Suárez, Analysis and Design of Autonomous Microwave Circuits. IEEE-Wiley, Hoboken, NJ, Jan. 2009.

[8] J. Jugo, J. Portilla, A. Anakabe, A. Suárez, J. M. Collantes, "Closed-loop stability analysis of microwave amplifiers," IEE Electronics Letters, vol. 37, no. 4, pp. 226-228, Feb. 2001.

[9] A. M. Elfrgani, R. G. Rojas, Stabilizing non-Foster circuits for electrically small antennas, AP-URSI, pp. 464-465, Jul. 2014.

[10] A. Suárez, S. Jeon, D. Rutledge, "Stability analysis and stabilization of power amplifiers," IEEE Microwave Magazine, vol. 7, pp. 51-65, Oct., 2006.

[11] F. Ramírez, M. Pontón, S. Sancho, A. Suárez, "Stability analysis of oscillation modes in quadruple-push and Rucker's oscillators," IEEE Trans. Microw. Theory Techn., vol. 56, no. 11, pp. 2648-2661, Nov., 2008.

[12] S. Sancho, F. Ramírez, A. Suárez, "General stabilization techniques for microwave oscillators”, IEEE Microw. Wireless Compon. Lett., vol. 15, no. 12 , pp. $868-870$, Dec. 2005. 